\title{
Impacto dos nutrientes na saúde óssea: novas tendências
}

\author{
Nutrients impact on bone health: new trends
}

\author{
Glaucia Queiroz Morais ${ }^{1}$, Maria Goretti Pessoa de Araújo Burgos²
}

\section{RESUMO}

A nutrição é fator importante modificável no desenvolvimento/manutenção da massa óssea (MO) e prevenção da osteoporose. Esta é uma doença caracterizada por decréscimo na massa esquelética e elevação da suscetibilidade a fraturas. O desenvolvimento do pico de massa óssea (PMO) é ainda o maior determinante dessa condição. Por isso, alterações com a idade sinalizam a importância da manutenção de nutrição adequada durante o desenvolvimento desse pico. Indivíduos que atingiram elevado PMO terão, na idade adulta, baixo risco de desenvolver doenças osteometabólicas com o envelhecimento. Os efeitos dos nutrientes sobre as estruturas esqueléticas são intensos e amplos; alguns, há anos, são consistentemente relacionados com a saúde óssea, como o cálcio, fósforo e vitamina D. Entretanto, outros nutrientes vêm sendo sugeridos como essenciais para o metabolismo ósseo normal: proteínas, lipídeos, potássio, vitaminas $\mathrm{K}$ e A e cloreto de sódio. A influência das interações entre tais fatores nutricionais, meio ambiente, estilo de vida e hereditariedade auxiliarão na compreensão da complexidade do desenvolvimento da osteoporose e subseqüentes fraturas. Em adição, déficit energético pode acarretar redução da MO, em face dos distúrbios endócrino-metabólicos desencadeados pela privação alimentar. Esta revisão objetiva elucidar o impacto dos nutrientes e

1. Especialista em Nutrição Clínica; Nutricionista do Setor de Traumato-Ortopedia do Hospital das Clínicas da Universidade Federal de Pernambuco UFPE - Recife (PE), Brasil.

2. Doutora em Nutrição; Nutricionista pesquisadora do Hospital das Clínicas da Universidade Federal de Pernambuco - UFPE - Recife (PE), Brasil.

Endereço para correspondência: Rua Baltazar Pereira, 70/601, Boa Viagem 51011-550 - Recife (PE) - Brasil. Tel.: (81) 3325-3873; fax: (81) 34231907.

E-mail: glauciaqm@ @otmail.com

Copyright RBO2007

Recebido em 25/10/06. Aprovado para publicação em 27/7/07. ingestão calórica sobre o osso, em diferentes estágios da vida, descrevendo possíveis interações entre nutrientes e como estas poderiam afetar a homeostase óssea e mineral.

Descritores - Osteoporose/prevenção \& controle; Fraturas óoseas/ prevenção \& controle; Nutrientes; Necessidades nutricionais; Micronutrientes; Bebida gasosa

\section{ABSTRACT}

Nutrition is an important factor in bone mass development/maintenance and in osteoporosis prevention. This disease is characterized by decreased skeletal mass and increased susceptibility to fractures. The greatest determinant of this condition is the peak bone mass (PBM). Hence, alterations over time indicate the importance of maintaining adequate nutrition during the development of this peak. In adult age, individuals who reached a high PBM will have a low risk of developing bone metabolic diseases when aging. Nutrients exert intense and wide-ranging effects on skeletal structures. Some of them have been consistently related with bone health, such as calcium, phosphorus and vitamin D. However, it has been suggested that other nutrients are essential for normal bone metabolism: proteins, lipids, potassium, vitamins $K$ and $A$, and sodium chloride. The influence of interactions between these nutritional factors, environment, lifestyle, and heredity will help understand the complex development of osteoporosis and subsequent fractures. In addition, energy deficits can lead to reduced bone mass, in view of endocrine-metabolic disorders caused by food deprivation. This review aims at clarifying the impact of nutrients and of calories intake on the bones during different stages in life, describing possible interactions among nutrients and how these could affect bone and mineral homeostasis.

Keywords - Osteoporosis/prevention \& control; Fractures, bonel prevention \& control; Nutrients; Nutritional requirements; Micronutrients; Carbonated beverages 


\section{INTRODUÇÃO}

O osso é um tecido dinâmico que passa por remodelações constantes durante a vida; além disso, forma um reservatório de cálcio $(\mathrm{Ca})$, magnésio $(\mathrm{Mg})$, fósforo $(\mathrm{P})$ e outros íons essenciais às funções homeostáticas ${ }^{(1)}$. A abordagem nutricional para alcance de MO máxima requer dieta balanceada e ingestão calórica adequada ${ }^{(2-3)}$. Os nutrientes relacionados são Ca, $\mathrm{P}$, proteína ${ }^{(4)}$, potássio $(\mathrm{K})$, vitaminas A e $\mathrm{K}$, flúor, zinco, cobre e boro ${ }^{(5)}$. Esta revisão objetiva elucidar o impacto dos nutrientes e ingestão calórica sobre o osso, em diferentes estágios da vida, descrevendo interações entre nutrientes e suas implicações sobre a homeostase óssea.

\section{PAPEL DOS MACRONUTRIENTES NA SAÚDE ÓSSEA}

I - Lipídeos - O risco de osteopenia, osteoporose e fraturas osteoporóticas é significantemente elevado em pacientes com alto percentual de gordura corpórea, independente do peso corpóreo, atividade física e idade ${ }^{(6)}$. Dados epidemiológicos sugerem que dietas ricas em gorduras, especialmente saturadas, podem contribuir para a redução da densidade mineral óssea (DMO) e elevação do risco de fraturas, na população jovem e idosa ${ }^{(7)}$. Segundo Corwin et al, dados do NHANHES III mostraram que o excesso de gordura foi negativamente associado com o conteúdo mineral ósseo e DMO em vários locais do esqueleto e em diferentes idades ${ }^{(8)}$.

A gordura pode reduzir a absorção intestinal de $\mathrm{Ca}$ e, possivelmente, elevar a excreção renal ${ }^{(9)}$. Dieta hiperlipídica aumenta a excreção intestinal e urinária de $\mathrm{Ca}$ e forma sabões insolúveis de $\mathrm{Ca}$ no intestino, resultando no aumento da sua excreção fecal ${ }^{(8)}$.

Muitos hormônios são críticos para o turnover ósseo; sobretudo o hormônio do crescimento (GH), envolvido na regulação óssea. O excesso de lipídeos pode inibir a secreção de GH, pelo aumento nos níveis de ácidos graxos plasmáticos livres $^{(10-11)}$.

Os ácidos graxos $\omega$-3 e $\omega$-6 são convertidos em vários precursores de eicosanóides (PGE1 e PGE2), importantes moduladores da saúde óssea. A PGE2 é um contribuinte do processo pró-inflamatório e elevadas concentrações podem inibir a formação óssea ${ }^{(9)}$.

Com o avançar da idade, o sistema de formação/reabsorção óssea torna-se desgastado, resultando em perda de tecido ósseo e aumento da gordura marrom. O excesso de gordura dietética pode exacerbar este desgaste por inibição da formação de osteoblastos maturos e aumento da adipogênese ${ }^{(10)}$. Entretanto, não existem, ainda, recomendações de lipídeos específicos que possam garantir maximização da MO.

II - Proteínas - A proteína é relacionada com crescimento e manutenção óssea, prevenindo a osteoporose ${ }^{(5)}$. Dietas hiperprotéicas poderiam ser deletérias à saúde óssea ${ }^{(11)}$; por outro lado, baixa ingestão protéica afetaria negativamente a saúde óssea ${ }^{(12)}$. Incremento na dieta de $50 \mathrm{~g}$ de proteína eleva aproximadamente $1,6 \mathrm{mmol}$ na excreção de $\mathrm{Ca}$, sendo a proteína um regulador do Ca urinário mais importante que a própria ingestão de $\mathrm{Ca}^{(11)}$. Kerstetter et al sugerem que dieta hiperprotéica afeta negativamente a retenção de $\mathrm{Ca}$, somente quando este tem sua ingestão inadequada ${ }^{(12)}$.

É consenso na literatura que a MO é a fonte adicional de Ca na urina, durante o consumo de dietas hiperprotéicas ${ }^{(13)}$. Isso resultaria em perda anual de 1 a $2 \%$ de MO na mulher adulta jovem, equivalente à perda estimada na menopausa preco$\mathrm{ce}^{(14)}$. É sugerido que dietas hiperprotéicas (origem animal) poderiam induzir a perda óssea por indução de acidose metabólica no organismo ${ }^{(15)}$. Como as proteínas animais são ricas em aminoácidos sulfurados, íons de amônia produzidos a partir desses aminoácidos levariam à redução do $\mathrm{pH}$ sanguíneo, ativando a reabsorção óssea que, em decorrência, levaria à perda de carbonato e citrato de Ca ósseo, mobilizados para neutralizar esse excesso de ácidos ${ }^{(15-16)}$. O mecanismo renal representa o principal elemento compensatório para a acidose e, no idoso, essa proteção renal pode ser incompleta, podendo o osso ser usado para neutralizar essa carga ácida ${ }^{(13)}$. Por outro lado, restrição aguda de proteína reduz a absorção de $\mathrm{Ca}$, com conseqüente hipocalcemia ${ }^{(13,17)}$. Em idosos com fraturas de quadril, frequientemente é observada ingestão hipoprotéica ${ }^{(11)}$.

O Institute of Medicine reportou nas últimas DRIs (2002) que há insuficientes evidências para o estabelecimento de ingestão máxima tolerável para a proteína dietética ${ }^{(17)}$. Entretanto, há concordância que dietas com teor protéico de 1,0 a $1,5 \mathrm{~g} / \mathrm{kg}$ peso/dia são associadas com metabolismo de Ca normal, não afetando a homeostase esquelética ${ }^{(11,18-19)}$.

Quanto à origem protéica, dados epidemiológicos não suportam a superioridade da vegetal sobre o esqueleto e estudos correlacionando proteína animal, vegetal e DMO não evidenciam benefícios em vegetarianos ${ }^{(20)}$. O excesso de proteína animal ou vegetal pode ser prejudicial à saúde óssea, mas esses efeitos podem ser modificados por outros nutrientes presentes na dieta, como o Ca, $\mathrm{K}$ e $\mathrm{P}^{(16)}$. 


\section{INGESTÃO CALÓRICA E SAÚDE ÓSSEA}

O impacto do déficit calórico e da perda de peso pode acarretar redução da MO e mudanças na composição corpórea ${ }^{(21-}$ 22). Doenças como anorexia e bulimia nervosa relacionam-se freqüentemente com osteopenia, osteoporose e maior vulnerabilidade para fraturas ${ }^{(23)}$. A perda óssea que ocorre em pacientes de baixo peso está ligada à deterioração do colágeno formador da matriz orgânica óssea, bem como ao gradual desequilíbrio com o processo de remodelação; idosos são mais propensos à perda óssea durante a redução de peso ${ }^{(24)}$. Enquanto, em mulheres, isso é devido à redução da ingestão dietética de $\mathrm{Ca}$ e/ou redução da eficiência da absorção, além de que, nelas, a deficiência estrogênica pós-menopausa aumenta a sensibilidade do esqueleto aos efeitos reabsortivos do $\mathrm{PTH}^{(22,}$ 25).

Obesos submetidos a regimes de acentuada perda de peso têm a saúde óssea em risco, pela restrição da ingestão de alimentos e Ca. A ingestão de $\mathrm{Ca}$, associada com elevada ingestão protéica e atividade física, desponta como um recurso preventivo para minimizar ou evitar essa perda óssea ${ }^{(26)}$. Entretanto, pesquisas adicionais serão necessárias para melhor elucidar os mecanismos que influenciam a densidade e qualidade óssea de vários locais vulneráveis a fraturas, durante a perda de peso $^{(25)}$.

\section{PAPEL DOS MINERAIS NA SAÚDE ÓSSEA}

I - Cálcio - A saúde óssea depende diretamente da ingestão regular de Ca na infância e adolescência; isso elevaria o PMO e reduziria o risco de osteoporose décadas mais tarde ${ }^{(27)}$. Necessidades dietéticas variam nos estágios da vida, sendo maiores durante o rápido crescimento de crianças/adolescentes, gestação/lactação e envelhecimento ${ }^{(11)}$. Vários eventos concomitantes concorrem para utilização do Ca pelo organismo, como: capacidade de absorção intestinal, metabolismo ósseo, excreção renal; ingestão de vitamina $\mathrm{D}$, elemento necessário à obtenção dos benefícios nutricionais do $\mathrm{Ca}^{(28)}$. Ingestão reduzida faz com que o mineral já utilizado no osso seja remanejado para outras funções fisiológicas vitais, como manutenção da calcemia ${ }^{(26)}$.

Na prevenção da osteoporose são utilizados produtos lácteos que, além de serem fontes de Ca de elevada disponibilidade, são ainda fontes de proteínas, lipídeos, sódio, K, fósforo, zinco, vitaminas A e B, contendo também componentes funcionais, como a proteína básica do leite (PBL) ${ }^{(29)}$. Esta é detentora de componentes capazes de promover a formação óssea e inibir a reabsorção ${ }^{(30)}$.
Para melhor aproveitamento do Ca dietético, deve-se evitar a ingestão concomitante de excesso de alimentos fontes de ácido oxálico; estes formam complexos com o $\mathrm{Ca}$, tornandose precipitados no lume intestinal em conseqüência do meio alcalino e, conseqüentemente, são excretados pelas fezes ${ }^{(31)}$. Do mesmo modo, fitatos podem complexar-se com o $\mathrm{Ca}$, situação que pode ocorrer com dietas muito ricas em fibras ${ }^{(32)}$.

Em resumo, o consumo de dietas que atendam às recomendações de Ca (através das DRIs), nos quais este nutriente esteja biodisponível, deve ser incentivado como estratégia primária na prevenção da osteoporose ${ }^{(33)}$.

II - Fósforo - O P é regulador da formação óssea e inibidor da reabsorção; contudo, há concordância de que altas quantidades poderiam ser prejudiciais para o osso ${ }^{(34)}$. Elevação no $\mathrm{P}$ dietético incrementa sua concentração plasmática, produzindo queda transitória no Ca plasmático ionizado, resultando em elevação da secreção do PTH e, potencialmente, reabsorção óssea ${ }^{(35)}$. Kawaura et al sugeriram que ingestão superior a $1.000 \mathrm{mg} /$ dia de $\mathrm{Ca}$ e relação $\mathrm{Ca} / \mathrm{P}$ superior a 0,74 foram associados com melhores valores de DMO em mulheres jovens $^{(33)}$. Pesquisa realizada por Koshihara evidenciou resultados que sugeriam que a elevação da razão dietética $\mathrm{Ca} / \mathrm{P}$ pode inibir a perda óssea e elevar a absorção intestinal de $\mathrm{Ca}$ em ratos $^{(35)}$.

Nas proteínas animais, o P é encontrado na forma de fosfato, sendo liberado durante a digestão; em contraste, muito do $\mathrm{P}$ vegetal é encontrado na forma de fitato, de difícil digestão e, por isso, pouco fósforo é absorvido. Deve-se levar em consideração, no momento do planejamento dietético, não somente a proporção $\mathrm{Ca} / \mathrm{P}$ da dieta, como também a origem (animal ou vegetal) do $\mathrm{P}$ dietético para prevenção/tratamento da osteoporose ${ }^{(36)}$.

Bebidas carbonatadas - Estudos mostram diminuição da MO e risco elevado de fraturas associados ao uso de bebidas carbonatadas ${ }^{(37)}$, enquanto outros não evidenciam tal relação ${ }^{(38)}$. Bebidas à base de colas contêm cafeína e ácido fosfórico, podendo afetar negativamente a saúde óssea, por meio da geração de carga ácida, no organismo; esta é causada pelo ácido fosfórico usado como acidulante nessas bebidas ${ }^{(39)}$. Nenhuma relação significativa foi observada com bebidas carbonatadas sem colas e DMO, porém, pesquisas adicionais são necessárias para confirmação desses $\operatorname{achados}^{(40)}$.

III - Potássio - Ingestão de frutas e vegetais pode contrabalançar o excesso de ácidos gerados por dietas hiperprotéi- 
cas, como resultado da promoção de bases do $\mathrm{K}^{(40)}$. Segundo Macdonald et al, a ingestão do $\mathrm{K}$ de fontes dietéticas pode influenciar positivamente os marcadores de saúde óssea, contribuindo, assim, para redução da osteoporose ${ }^{(40)}$. Ao mesmo tempo, Rafferty et al relataram que a ingestão dietética de $\mathrm{K}$ poderia reduzir a excreção urinária de $\mathrm{Ca}$ e, conseqüentemente, melhorar seu balanço ${ }^{(41)}$. A habilidade do $\mathrm{K}$ em reduzir a excreção urinária de Ca deve-se ao seu conteúdo em sais álcalis, os quais reduzem a acidose endógena, elevando o $\mathrm{pH}$ sanguíneo e concentração plasmática de bicarbonato ${ }^{(42)}$.

IV - Cloreto de sódio ( $\mathbf{N a C l )}-\mathrm{O} \mathrm{NaCl}$ dietético representa prejuízo em potencial para a MO; com aumento da sua ingestão, observa-se elevação da hipercalciúria ${ }^{(43)}$. A reabsorção renal de $\mathrm{Ca}$ é diretamente proporcional à reabsorção de sódio (Na) e, ao elevar-se o $\mathrm{NaCl}$, a reabsorção fracional de $\mathrm{Na}$ é diminuída, ocasionando redução paralela na absorção de $\mathrm{Ca}$ e prejuízos à $\mathrm{MO}^{(44)}$. Carbone et al sugeriram que, em mulheres na pós-menopausa, dieta restrita em $\mathrm{Na}$ ( $2 \mathrm{~g} / \mathrm{dia})$ poderá ser benéfica para a $\mathrm{DMO}^{(44)}$.

\section{PAPEL DAS VITAMINAS NA SAÚDE ÓSSEA}

I - Vitamina A - A ingestão excessiva de retinol proveniente da dieta ou suplementos tem associação negativa com a $\mathrm{DMO}^{(45)}$. Do mesmo modo, concentrações plasmáticas elevadas de retinol são relacionadas com alta incidência de fraturas de quadril ${ }^{(46)}$. Por outro lado, não há evidências relacionando danos com ingestões de betacaroteno ${ }^{(46)}$. Segundo Genaro et al, elevada ingestão a longo prazo de vitamina A estimula a reabsorção óssea e inibe a sua formação ${ }^{(46)}$. Lind $e t$ al mostraram, em estudo com ratos, que a hipervitaminose A subclínica causa fragilidade óssea, possivelmente devido a interações da vitamina A com outras vitaminas lipossolúveis ${ }^{(47)}$. A subtoxicidade sem um sinal clínico de toxicidade é relevante, pelo fato de a ingestão de fontes pré-formadas freqüentemente exceder o limite das RDA para adultos ${ }^{(48)}$. Porém, dados evidenciam correlação positiva entre consumo de frutas/vegetais (ricos em carotenóides) e saúde óssea ${ }^{(49)}$.

A vitamina A é necessária para o crescimento ósseo normal; entretanto, ingestões acima de $1.500 \mu /$ dia de retinol foram associadas a menores DMO e maiores riscos de fraturas nas populações americanas e suecas ${ }^{(50)}$. Mesmo assim, não é possível determinar níveis seguros de ingestões adequadas de vitamina $\mathrm{A}$, acima dos quais a saúde óssea seria comprometi$\mathrm{da}^{(45)}$.

II - Vitamina $\mathbf{K}$ - A vitamina K é um nutriente essencial para a coagulação sanguínea; além disso, funciona como co- fator na carboxilação de várias proteínas ósseas. A deficiência dessa vitamina pode reduzir a DMO e elevar o risco de osteoporose/fraturas osteoporóticas ${ }^{(51)}$. Suas formas naturais, a K1 (filoquinona) e K2 (menadiona), são consideradas protetores potenciais contra a incidência da osteoporose ${ }^{(52)}$. Apesar disso, Rejnmark et al não verificaram nenhuma associação entre ingestão de vitamina K1 e DMO ou risco de fraturas em mulheres na pré-menopausa ${ }^{(53)}$. Baixas concentrações plasmáticas de vitamina $\mathrm{K}$ são relatadas em pacientes com osteoporose e a osteocalcina plasmática parece estar descarboxilada nesses indivíduos. A osteocalcina descarboxilada é um significante fator de risco para fraturas de quadril, porém, mais pesquisas, sobretudo em humanos, se tornam necessárias para elucidar tais mecanismos ${ }^{(54)}$.

III - Vitamina D - A vitamina D é essencial para a homeostase do $\mathrm{Ca}$ e $\mathrm{P}$, além de necessária para o desenvolvimento/manutenção normal das estruturas esqueléticas ${ }^{(55)}$. Pesquisas recentes sugerem que a vitamina $\mathrm{D}$ ativa pode prevenir fraturas, não somente pela elevação da absorção intestinal de $\mathrm{Ca}$, mas também por melhorar a qualidade e/ou resistência óssea ${ }^{(55)}$.

Deficiência subclínica de vitamina $\mathrm{D}$ em indivíduos adultos estabelece-se de forma sutil, com hipocalcemia leve, hiperparatireoidismo reacional e perda de $\mathrm{MO}^{(56-57)}$. Deficiência grave poderá causar raquitismo, osteomalácia e exacerbar a perda óssea na osteoporose ${ }^{(57)}$. Metanálise realizada por Bischoff-Ferrari et al mostrou que suplemento de colecalciferol (700-800UI/dia) parece reduzir o risco de fraturas de quadril e fraturas vertebrais em idosos institucionalizados ${ }^{(58)}$.

Recentes recomendações sugerem que, na ausência de exposição solar adequada, adultos necessitariam ingerir 1.000UI de vitamina $\mathrm{D} 3 / \mathrm{dia}^{(59)}$. Contudo, ainda não há consenso na literatura acerca da ingestão alimentar apropriada de vitamina $\mathrm{D}$, por grupo racial, o que seria adequado para prevenção de fraturas ósseas ${ }^{(60)}$.

\section{COMENTÁRIOS}

O consumo adequado dos nutrientes envolvidos no metabolismo ósseo pode prevenir ou reduzir a incidência de doenças ósseas, dentre elas, a osteoporose. O PMO e DMO ideais mostram correlação positiva com ingestão de nutrientes específicos, sobretudo nos grupos mais vulneráveis à perda óssea. Diante desses achados, é imperioso direcionar esforços no sentido de conscientizar profissionais de saúde para o aperfeiçoamento de medidas dietéticas que possam prevenir e/ou reduzir essas alterações. 


\section{REFERÊNCIAS}

1. Holick MF, Krane SM. Introdução ao metabolismo ósseo e mineral. In: Braunwald E, Fauci AS, Kasper DL, Hauser SL, Longo DL, Jameson JL, editores. Harrisson Medicina Interna. 15a. ed. Rio de Janeiro: McGrawHill; 2002. p. 2329-43.

2. Amaya-Farfan J. Fatores nutricionais que influem na formação e manutenção do osso. Rev Nutr PUCCAMP.1994;7(2):148-72.

3. Miggiano GA, Gagliardi L. [Diet, nutrition and bone health]. Clin Ter. 2005;156(1-2):47-56. Italian.

4. Teegarden D, Lyle RM, McCabe GP, McCabe LD, Proulx WR, Michon $\mathrm{K}$, et al. Dietary calcium, protein, and phosphorus are related to bone mineral density and content in young women. Am J Clin Nutr. 1998; 68(3):749-54. Comment in: Am J Clin Nutr. 1998;68(3):523-4.

5. Ilich JZ, Kerstetter JE. Nutrition in bone health revisited: a story beyond calcium. J Am Coll Nutr. 2000;19(6):715-37.

6. Strewler G. Mineral metabolism and metabolic bone disease. In: Greenspan F, Strewler G, editors. Basic \& clinical endocrinology. New York: Prentice-Hall; 1997. p. 263-316.

7. Raisz LG, Rodan GA. Pathogenesis of osteoporosis. Endocrinol Metab Clin North Am. 2003;32(1):15-24. Review.

8. Corwin RL, Hartman TJ, Maczuga SA, Graubard BI. Fat intake and bone health in NHANES III. FASEB J. 2002;16:A625.

9. Corwin RL. Effects of dietary fats on bone health in advanced age. Prostaglandins Leukot Essent Fatty Acids. 2003;68(6):379-86. Review.

10. Kerstetter JE, O'Brien KO, Insogna KL. Dietary protein, calcium metabolism, and skeletal homeostasis revisited. Am J Clin Nutr. 2003; 78(3 Suppl):584S-592S. Review.

11. Bonjour JP. Dietary protein: an essential nutrient for bone health. J Am Coll Nutr. 2005;24(6 Suppl):526S-36S.

12. Kerstetter JE, Allen LH. Protein intake and calcium homeostasis. Adv Nutr Res. 1994;9:167-81. Review.

13. Rosen CJ, Kiel DP. The aging skeleton. In: Favus MJ, editor. Primer on the metabolic bone diseases and disorders of mineral metabolism. 4th ed. Philadelphia: Lippincott Williams \& Wilkins; c1999. p. 57-9.

14. Munger RG, Cerhan JR, Chiu BC. Prospective study of dietary protein intake and risk of hip fracture in postmenopausal women. Am J Clin Nutr. 1999;69(1):147-52. Comment in: Am J Clin Nutr. 1999;70(2):303. Am J Clin Nutr. 2001;73(5):990-2.

15. Zemel MB. Calcium utilization: effect of varying level and source of dietary protein. Am J Clin Nutr. 1998;48(3 Suppl):880-3.

16. Barzel US, Massey LK. Excess dietary protein can adversely affect bone. J Nutr. 1998;128(6):1051-3. Review.

17. Institute of Medicine. Dietary reference intakes: protein. Washington (DC): National Academy Press; 2002.

18. Massey LK. Dietary animal and plant protein and human bone health: a whole foods approach. J Nutr. 2003;133(3):862S-865S.

19. Reed JA, Anderson JJ, Tylavsky FA, Gallagher PN. Comparative changes in radial-bone density of elderly female lacto-ovovegetarians and omnivores. Am J Clin Nutr. 1994;59(5 Suppl):1197S-1202S. Erratum in: Am J Clin Nutr 1994;60(6):981.

20. Holick M. Vitamin D: importance in prevention of cancers, type I diabetes, heart disease, and osteoporosis. Am J Clin Nutr. 2004;79(3): 362-71. Review. Erratum in: Am J Clin Nutr. 2004;79(5):890.

21. Freitas BSA, Carvalho CR. Importantes mecanismos de perda da massa óssea nos transtornos de alimentação. Rev Bras Nutr Clin. 2006;21(2):14954.
22. Grinspoon S, Thomas E, Pitts S, Gross E, Mickley D, Miller K, et al Prevalence and predictive factors for regional osteopenia in women with anorexia nervosa. Ann Intern Med. 2000;133(10):790-4. Comment in: Ann Intern Med. 2000;133(10):828-30. Ann Intern Med. 2001;135(9): 843-4.

23. Shils ME, Olson JA, Shike M, Ross AC. Tratado de nutrição moderna na saúde e na doença. 9a ed. São Paulo: Manole; 2003.

24. Shapses SA, and Riedt CS. Bone, body weight, and weight reduction: what are the concerns? J Nutr. M 2006;136(6):1453-6.

25. Bowen J, Noakes M, Clifton PM. A high dairy protein, high-calcium diet minimizes bone turnover in overweight adults during weight loss. J Nutr. 2004;134(3):568-73.

26. Montilla RNG, Aldrighi JM, Marucci MFN. Relação cálcio/proteína da dieta de mulheres no climatério. Rev Assoc Med Bras (1992). 2004;50(1): $52-4$.

27. Flynn A. The role of dietary calcium in bone health. Proc Nutr Soc. 2003; 62(4):851-8.

28. Uenishi, K. [Prevention of osteoporosis by foods and dietary supplements. Prevention of osteoporosis by milk and dairy products]. Clin Calcium. 2006;16(10):1606-14. Japanese

29. Itabashi A. Prevention of osteoporosis by foods and dietary supplements. Milk basic protein (MBP) increases bone mineral density in young adult women and perimenopausal women. Clin Calcium. 2006;16(10):1632-8. Japanese.

30. Coelho RG. Interações nutricionais. Rev Metab Nutr. 1995;2(3):106-17.

31. Haack VS, Chesters JG, Vollendorf NW, Story JA, Marlett JA. Increasing amounts of dietary fiber provided by foods normalizes physiologic response of the large bowel without altering calcium balance or fecal steroid excretion. Am J Clin Nutr. 1998;68(3):615-22.

32. Lanzillotti HS, Lanzillotti RS, Trotte APR, Dias AS, Bornand B, Costa EAMM. Osteoporose em mulheres na pós-menopausa, cálcio dietético e outros fatores de risco. Rev Nutr. 2003;16(2):181-93.

33. Kawaura A, Nishida Y, Takeda E. [Phosphorus intake and bone mineral density (BMD)]. Clin Calcium. 2005;15(9):1501-6. Japanese.

34. Calvo MS, Kumar R, Heath H. Elevated secretion and action of serum parathyroid hormone in young adults consuming high phosphorus, low calcium diets assembled from common foods. J Clin Endocrinol Metab. 1998;66(4):823-9.

35. Koshihara M, Masuyama R, Uehara M, Suzuki K. Effect of dietary calcium: Phosphorus ratio on bone mineralization and intestinal calcium absorption in ovariectomized rats. Biofactors. 2004;22(1-4):39-42.

36. Omi N, Ezawa I. [Phosphorus intake and osteoporosis]. Clin Calcium. 2001;11(10):1309-14. Japanese.

37. Kim SH, Morton DJ, Barrett-Connor EL. Carbonated beverage consumption and bone mineral density among older women: the Rancho Bernardo Study. Am J Public Health. 1997;87(2):276-9.

38. Petridou E, Karpathios T, Dessypris N, Simou E, Trichopoulos D. The role of dairy products and non alcoholic beverages in bone fractures among schoolage children. Scand J Soc Med. 1997;25(2):119-25.

39. Tucker KL, Morita K, Qiao N, Hannan MT,Cupples LA, Kiel DP. Colas, but not other carbonated beverages, are associated with low bone mineral density in older women: The Framingham Osteoporosis Study. Am J Clin Nutr. 2006;84(4):936-42.

40. Macdonald HM, New SA, Fraser WD, Campbell MK, Reid DM. Low dietary potassium intakes and high dietary estimates of net endogenous 
acid production are associated with low bone mineral density in premenopausal women and increased markers of bone resorption postmenopausal women. Am J Clin Nutr. 2005;81(4):923-33.

41. Rafferty K, Davies KM, Heaney RP. Potassium intake and the calcium economy. J Am Coll Nutr. 2005;24(2):99-106.

42. Chan EL, Ho CS, MacDonald D, Ho SC, Chan TY, Swaminathan R Interrelationships between urinary sodium, calcium, hydroxyproline and serum PTH in healthy subjects. Acta Endocrinol (Copenh). 1992;127(3): 242-5.

43. Nordin BE, Need AG, Morris HA, Horowitz M. The nature and significance of the relationship between urinary sodium and urinary calcium in women. J Nutr. 1993;123(9):1615-22.

44. Carbone LD, Barrow KD, Bush AJ, Boatright MD, Michelson JA, Pitts $\mathrm{KA}$, et al. Effects of a low sodium diet on bone metabolism. J Bone Miner Metab. 2005;23(6):506-13

45. Crandall C. Vitamin A intake and osteoporosis: a clinical review. J Womens Health (Larchmt). 2004;13(8):939-53. Review.

46. Genaro P de S, Martini LA. Vitamin A supplementation and risk of skeletal fracture. Nutr Rev. 2004;62(2):65-7.

47. Lind PM, Johansson S, Rönn M, Melhus H. Subclinical hypervitaminosis A in rat: measurements of bone mineral density (BMD) do not reveal adverse skeletal changes. Chem Biol Interact. 2006;159(1):73-80.

48. Penniston KL, Tanumihardjo SA. The acute and chronic toxic effects of vitamin A. Am J Clin Nutr. 2006;83(2):191-201. Review.

49. Wattanapenpaiboon N, Lukito W, Wahlqvist ML, Strauss BJ. Dietary carotenoid intake as a predictor of bone mineral density. Asia Pac J Clin Nutr. 2003;12(4):467-73

50. Michaelsson K, Lithell H, Vessby B, Melhus H. Serum retinol levels and the risk of fracture. N Engl J Med. 2003;348(4):287-94.
51. Adams J, Pepping J. Vitamin $\mathrm{K}$ in the treatment and prevention of osteoporosis and arterial calcification. Am J Health Syst Pharm. 2005; 62(15):1574-81.

52. Hosoi T. [Prevention of osteoporosis by foods and dietary supplements. Vitamin K and bone metabolism]. Clin Calcium. 2006;16(10):1651-4. Japanese.

53. Rejnmark L, Vestergaard P, Charles P, Hermann AP, Brot C, Eiken P, Mosekilde L. No effect of vitamin K1 intake on bone mineral density and fracture risk in perimenopausal women. Osteoporos Int. 2006;17(8): 1122-32.

54. Katagiri H. [Active vitamin D and vitamin $\mathrm{K}$ as therapeutic agents for osteoporosis]. Nippon Rinsho. 2006;64(9):1639-43. Japanese.

55. Cheskis BJ, Freedman LP, Nagpal S. Vitamin D receptor ligands for osteoporosis. Curr Opin Investig Drugs. 2006;7(10):906-11.

56. Premaor MO, Furlanetto TW. Hipovitaminose D em adultos: entendendo melhor a apresentação de uma velha doença. Arq Bras Endocrinol Metab. 2006;50(1):25-37.

57. Bandeira F, Griz L, Dreyer P, Eufrazino C, Bandeira C, Freese E. Deficiência de vitamina D: uma perspectiva global. Arq Bras Endocrinol Metab. 2006;50(4):640-6.

58. Bischoff-Ferrari HA, Willett WC, Wong JB, Giovannucci E, Dietrich T, Dawson-Hughes B. Fracture prevention with vitamin D supplementation: a meta-analysis of randomized controlled trials. JAMA. 2005;293(18): 2257-64. Comment in: ACP J Club. 2005;143(3):72-4.

59. Holick MF. The role of vitamin D for bone health and fracture prevention. Curr Osteoporos Rep. 2006;4(3):96-102.

60. Hathcock JN, Shao A, Vieth R, Heaney R. Risk assessment for vitamin D Am J Clin Nutr. 2007;85(1):6-18. Review. 